\title{
DEUX NOUVEAUX ISOPODES ABYSSAUX DE L'ATLANTIQUE NORD APPARTENANT AU GENRE IANIRELLA BONNIER, 1885 1)
}

PAR

\section{PIERRE CHARDY}

Centre Océanologique de Bretagne, B.P. 337, 29N, Brest, France

- Le genre Janirella Bonnier, 1885, compte de nombreux représentants en Atlantique Nord (J. nanseni Bonnier, 1885; J. abyssicola Richardson, 1911; J. glabra Richardson, 1911; J. spongicola Hansen, 1916; J. lobata Richardson, 1908; J. laevis Hansen, 1916; J. vemae Menzies, 1962; J. priseri Chardy, 1972) ainsi que dans les mers adjacentes ( $J$. bonnieri Stephensen, 1915 en Méditerranée et $J$. carribica Menzies, 1956 en mer des Caraïbes). A cette liste viennent s'ajouter deux espèces nouvelles, J. bocqueti sp. n. et J. laubieri sp. n., récoltées au cours de la campagne Noratlante du N.O. ,Jean Charcot". -

\section{Janirella bocqueti sp. n. ${ }^{2}$ )}

Matériel étudié. - Campagne „Noratlante”: août-octobre 1969: Prélèvement $\mathrm{n}^{\circ} 20$ - B04: $52^{\circ} 06,3^{\prime} \mathrm{N} 45^{\circ} 33,6^{\prime} \mathrm{W} ; 4166 \mathrm{~m}$; chalut Blake double perche. 2 우 은 - Prélèvement $\mathrm{n}^{\circ} 26-$ E04: $55^{\circ} 52,5^{\prime} \mathrm{N} 49^{\circ} 29,5^{\prime} \mathrm{W} ; 3465 \mathrm{~m}$; drague épibenthique. 2 of of (dont l'holotype) et 1 \% . L'holotype est déposé dans la collection du Muséum National d'Histoire Naturelle, Paris.

Description. - Le spécimen désigné comme holotype est un mâle adulte mesurant $3,9 \mathrm{~mm}$ de l'extrémité du rostre au bord postérieur du pléotelson. La plus grande largeur, mesurée entre les expansions latérales postérieures du 3ème péréionite est de $2,4 \mathrm{~mm}$.

Forme du corps (fig. 1A): Ovale, caractérisé par des prolongements latéraux épais et épineux, porteurs de nombreux poils. Rostre massif, légèrement plus long que large, orné de deux pointes latérales et deux pointes apicales terminées chacune par une épine. Face dorsale du céphalon pourvue d'une paire de tubercules spinescents. Péréionites I et II munis d'une épine médio-dorsale très discrète. Bords antérieurs et postérieurs des péréionites III à VII garnis d'une rangée de poils. Pléotelson plus large que long, pourvu de 4 dents latérales inégales terminées chacune par une épine apicale.

1) Contribution $n^{\circ} 88$ du Groupe Scientifique du Centre Océanologique de Bretagne. Résultats scientifiques de la campagne du N.O. Jean Charcot en Atlantique Nord (Août-Octobre 1969). Publication $\mathrm{n}^{\circ} 18$.

2) Cette espèce est très respectueusement dédiée au Professeur C. Bocquet, Paris. 


\section{JANIRELLA DE L'ATLANTIQUE NORD}

Antennule (fig. 2B): flagellum composé de 8 articles dont les cinq derniers portent chacun une longue soie sensorielle.

Antenne (fig. 2A): cassée au niveau du 4ème article chez l'holotype (ainsi que chez les paratypes). Présence d'un tubercule conique sur le bord externe du 3ème article, surmonté d'une épine distale.

Mandibule (fig. 1E): pourvue d'un palpe de 3 articles dont le dernier est terminé par une touffe de poils. Pars molaris massive, armée d'une couronne épineuse entourée de quelques soies.

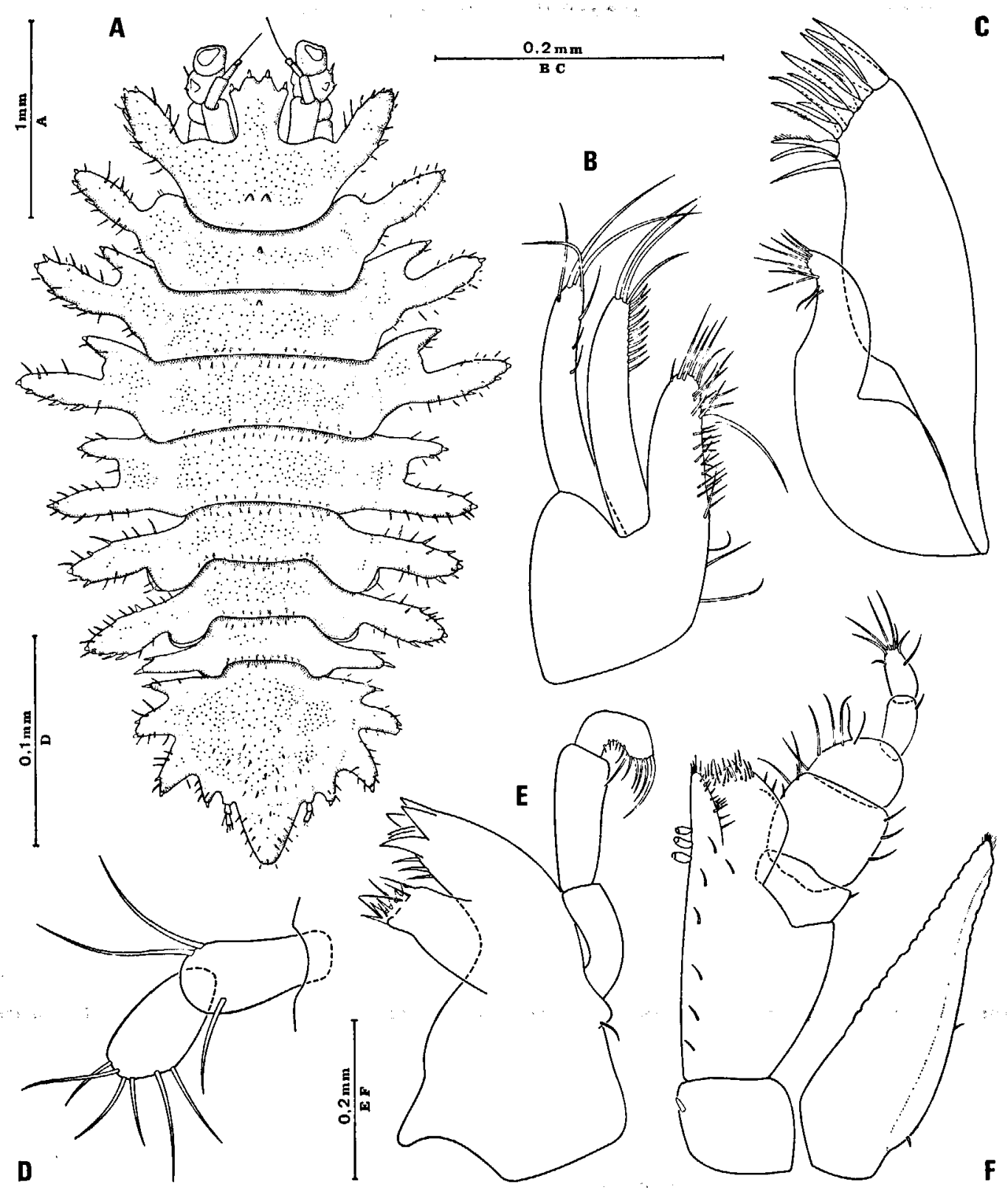

Fig. 1. Janirella bocqueti sp. n., holotype ( $\hat{\partial}$ ). A, vue dorsale de l'habitus; B, maxilles; C, maxillules; $D$, uropodes; $E$, mandibule gauche; $F$, maxillipède. 
Maxillules (fig. 1C): endite externe robuste porteur d'une rangée de fortes soies dentées. Endite interne réduit terminé par quelques soies fines.

Maxilles (fig. 1B): lobe inférieur large et court muni latéralement d'une longue soie à base renflée.

Maxillipèdes (fig. 1F): basipodite orné de 3 rétinacles sur son bord interne. Epipodite orné d'une touffe apicale de poils courts.

Péréiopodes: premier péréiopode (fig. 2D) robuste possédant un dactylopodite armé d'une forte griffe. Carpopodite porteur de 7 grosses épines sur son bord

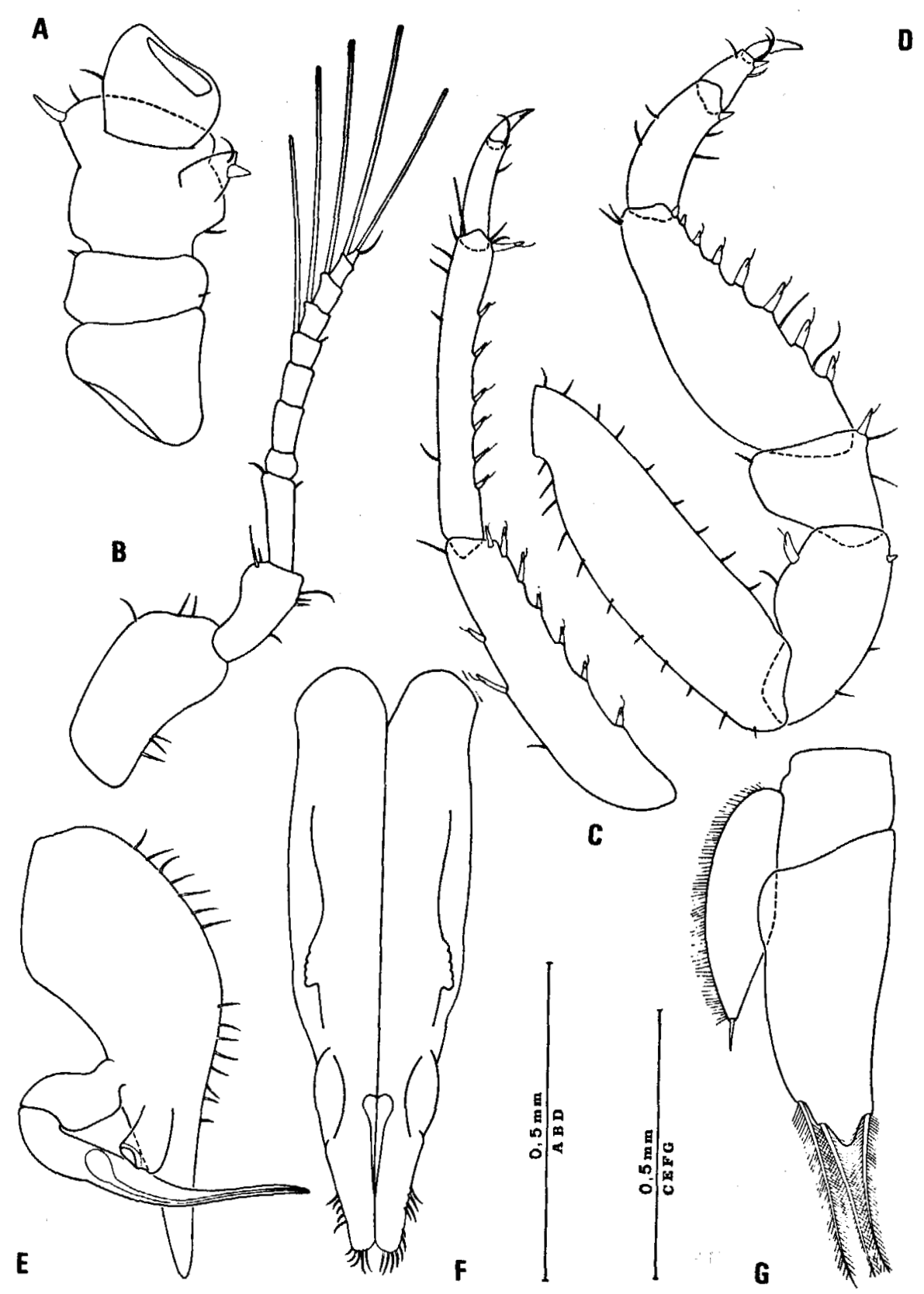

Fig. 2. Janirella bocqueti sp. n., holotype ( $(\hat{O})$. A, antenne; $B$, antennule; $C$, deuxième péréiopode; D, premier péréiopode; E, deuxième pléopode; F, premier pléopode; G, troisième pléopode. 


\section{JANIRELLA DE L'ATLANTIQUE NORD}

interne. Les autres péréiopodes, tous semblables (fig. 2C) sont beaucoup plus grèles.

Pléopodes: Les deux premiers pléopodes sont différenciés chez le mâle. L'appareil copulateur mâle fixé à la face interne du deuxième pléopode présente une tige chitineuse relativement courte et trapue.

Uropodes: courts, uniramés, composés de deux articles sensiblement de la même longueur (fig. 1D).

Discussion systematique. - La présence d'un pléotelson muni de quatre dents latérales nettement individualisées, permet d'isoler au sein du genre Janirella un groupe d'espèces comprenant J. nanseni Bonnier, 1896, J. bonnieri Stephensen, 1915, J. polychaeta Birstein, 1963, J. arnata Birstein, 1963, et J. spongicola Hansen, 1916, auxquelles se joint J. bocqueti sp. n. La présence d'un rostre massif à quatre pointes et la disposition latérale des quatre dents inégales du pléotelson caractérise $]$. bocqueti sp. n. au sein de ce groupe.

\section{Janirella laubieri sp. n. $^{3}$ )}

Matériel étudié. - Campagne „Noratlante”, août-octobre 1969: Prélèvement ${ }^{\circ} 63$ - E08: $36^{\circ} 48,5^{\prime} \mathrm{N} 45^{\circ} 33,6^{\prime} \mathrm{W} ; 3663 \mathrm{~m}$; drague épibenthique. 2 ô o (dont l'holotype) et 1 \%. L'holotype est déposé au Muséum National d'Histoire Naturelle, Paris.

Description. - L'holotype est un mâle adulte mesurant $4 \mathrm{~mm}$ de l'extrémité du rostre au bord postérieur du pléotelson. La largeur mesurée entre les expansions latérales postérieures du 3ème péréionite est de $2,3 \mathrm{~mm}$.

Forme du corps (fig. 3A): Aspect général du corps étroit, caractérisé par les prolongements latéraux des somites grèles et épineux. Absence de rostre céphalique. Face dorsale ornée de forts tubercules saillants dont le nombre est variable selon les segments (céphalon et péréionite I: 3 tubercules, péréionite II: 7; péréionite III-IV: 5; péréionite V-VII et telson: 2). Pléotelson plus long que large, pourvu de 3 dents latérales sub-égales terminées chacune par une forte épine.

Antennule (fig. 4B): flagellum composé de 7 articles dont les 4 derniers portent chacun une soie sensorielle.

Antenne (fig. 4A): bord interne du 3ème article du pédoncule orné d'un tubercule (tous les exemplaires ont les antennes brisées au niveau de la 4ème articulation).

Mandibule (fig. 3E): pourvue d'un palpe à 3 articles dont le second est porteur de deux fortes soies pennées. Pars molaris entourée d'un panache de soies fines.

Maxillules (fig. 3C): Endite interne bien développée terminée par une frange de poils raides.

Maxilles (fig. 3B): bord interne du lobe intermédiaire porteur d'une rangée de soies courtes et régulièrement insérées.

Maxillipèdes (fig. 3F) : basipodite orné de trois rétinacles sur son bord interne. Epipodite étroit dépourvu de poils.

3) Cette espèce est très amicalement dédiée à Lucien Laubier - Chef du Département scientifique du C.O.B. 
Péréiopodes: premier péréiopode (fig. 4G) doté d'un carpopodite large et robuste, armé d'une rangée d'épines de longueur inégale, sur son bord interne. Propodite étroit et allongé.

Pléopodes: premier pléopode (fig. 4E) formé d'une longue lamelle étroite bordée à son extrémité distale de quelques soies courtes. Deuxième pléopode (fig. $4 \mathrm{~F})$ porteur d'un appareil copulateur terminé par une longue tige chitineuse.

Uropodes (fig. 3D): courts, biarticulés; l'article basilaire est sensiblement plus long que l'article terminal.

Discussion systematique. - L'absence de rostre céphalique permet de distinguer facilement parmi les représentants du genre Janirella un groupe de cinq espèces:

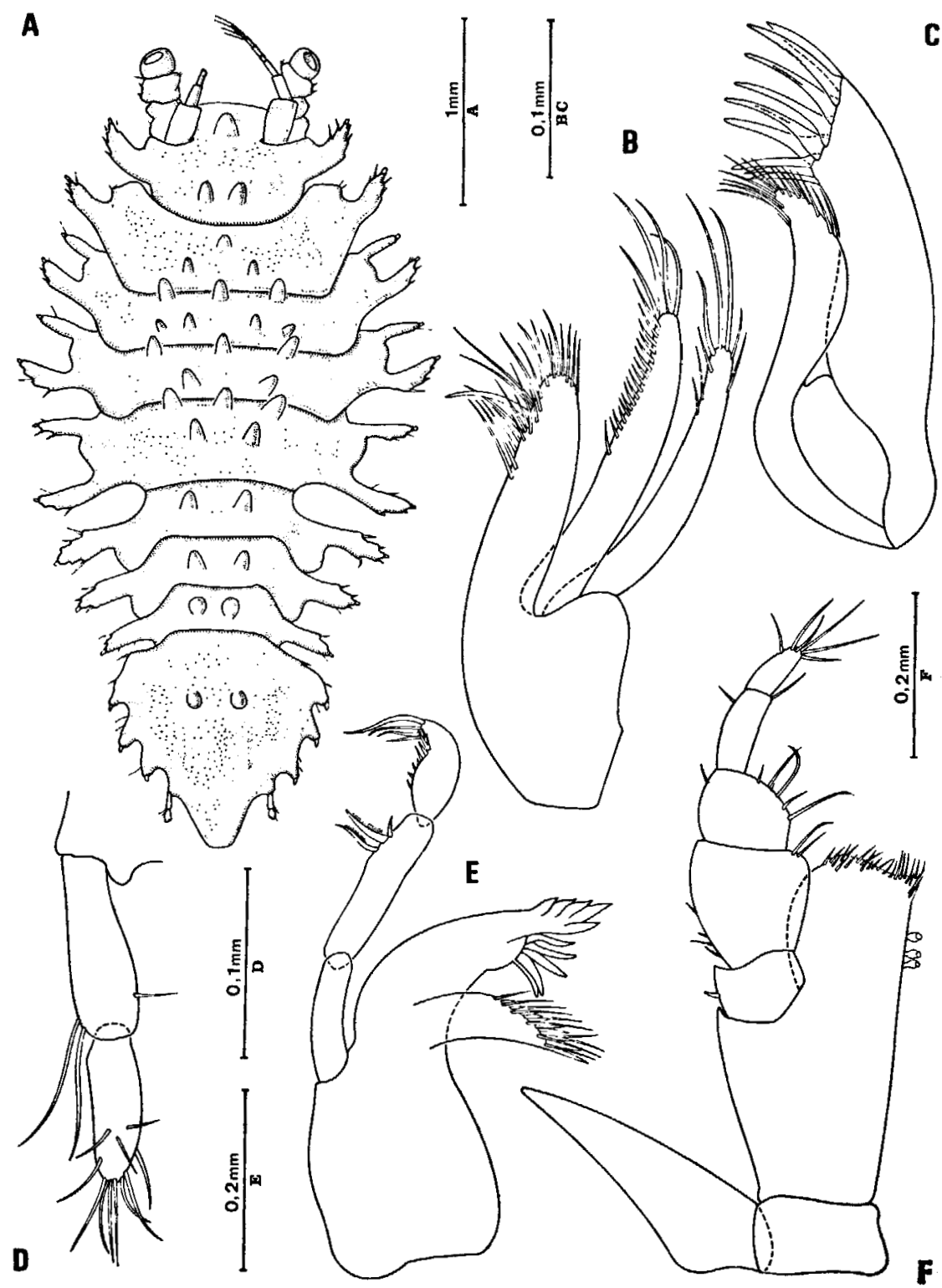

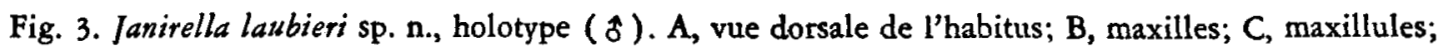
$D$, uropodes; E, mandibule gauche; $E$, maxillipède. 
J. vemae Menzies, 1962; J. bifida Menzies, 1962; J. erostrata Birstein, 1963; J. tuberculata Birstein, 1963 et J. laubieri sp. n.

Parmi ces espèces, seules $J$. tuberculata et $J$. laubieri sp. n. portent des tubercules sur la face dorsale des somites. Cependant les deux espèces ne peuvent être confondues: J. tuberculata possède de nombreux tubercules dorsaux de taille réduite, un flagellum antennulaire comportant 12 articles et un pléotelson dont l'extrémité est pointue, alors que $J$. laubieri sp. n. porte un nombre réduit de tubercules dorsaux de grande taille, un flagellum antennulaire de 7 articles et un pléotelson dont l'extrémité est courte et émoussée.

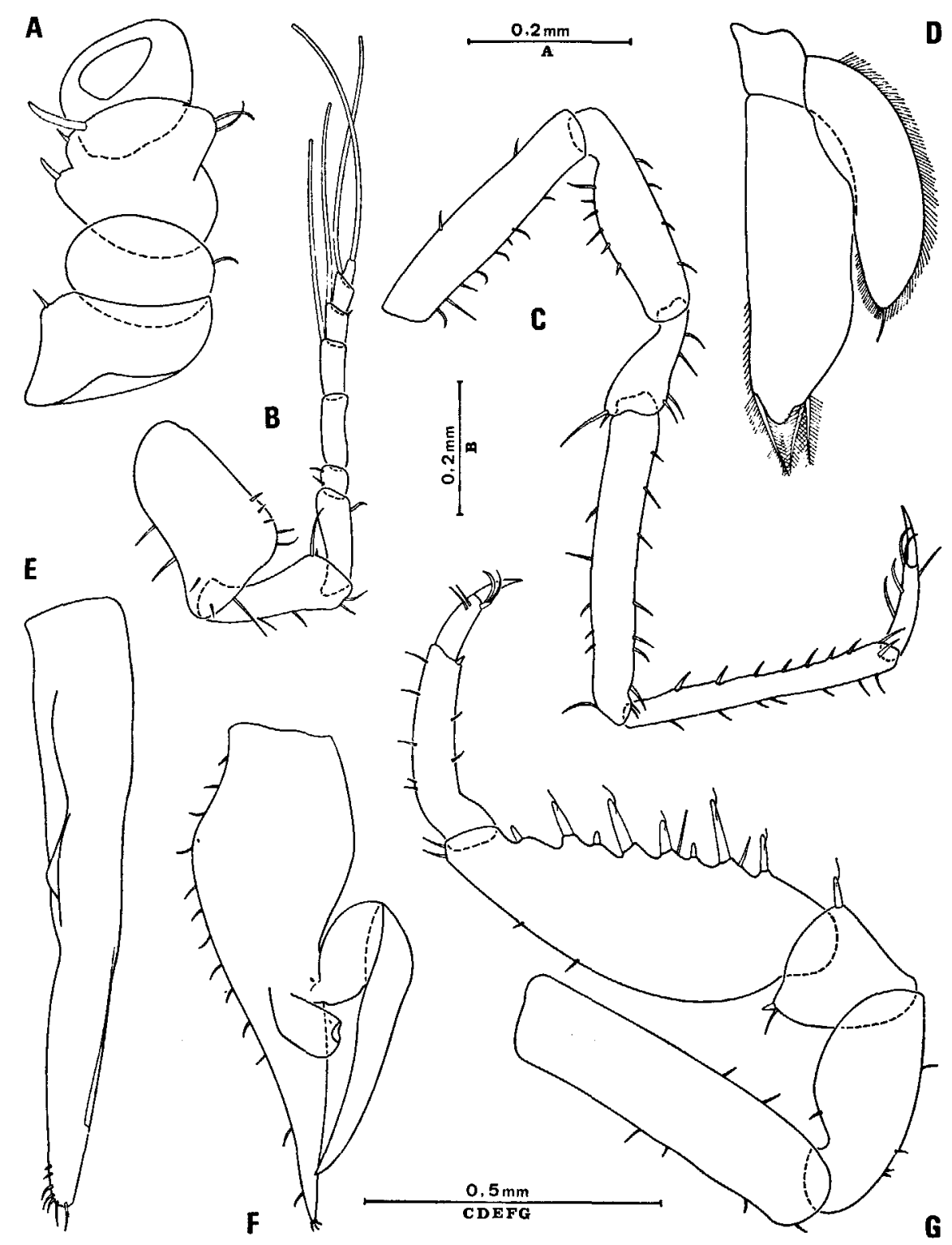

Fig. 4. Janirella laubieri sp. n. holotype ( ô ). A, antenne; B, antennule; C, deuxième péréiopode; D, troisième pléopode; $\mathrm{E}$, premier pléopode; $\mathrm{F}$, deuxième pléopode; $\mathrm{G}$, premier péréiopode. 


\section{CHARDY, JANIRELLA DE L'ATLANTIQUE NORD}

\section{SUMMARY}

Description of two new species of Janirella (Isopoda, Janirellidae) collected in North Atlantic Ocean from depths exceeding $3000 \mathrm{~m}$ during the "Noratlante" cruise of August-October 1969.

\section{REFERENCES BIBLIOGRAPHIQUES}

Birstein, Y. A., 1963. Isopods from the ultra-abyssal zone of the Bougainville Trench. Zool. Zh., 42: 814-833.

- 1963a. Deep-sea Isopods of the North Western Pacific: 1-213. (Institut Okeanologii, Akademia Nauk SSSR, Moskva).

Bonnier, J., 1896. Edriophthalmes. Résultats scientifiques de la campagne du „Caudan" dans le Golfe de Gascogne. Ann. Univ. Lyon, 26: 527-689.

Chardy, P., 1972. Janirella priseri sp. n., Isopode abyssal de l'Atlantique Nord. Crustaceana, (suppl.) 3: 11-18.

Hansen, H. J., 1916. Crustacea Malacostraca, 3. Dan. Ingolf Exped., 3 (5): 1-262.

Menzies, R. J. 1956. New abyssal tropical Atlantic isopods with observations on their biology. Amer. Mus. Notivates, 1798: 1-16.

1956a. New bathyal Isopoda from the Caribbean with observations on their nutrition. Breviora, 63: 1-10.

$\longrightarrow$, 1962. The isopods of abyssal depths in the Atlantic Ocean. Vema Research Ser., 1: 79-206.

RICHARDSON, H., 1908. Some new isopods of the superfamily Aselloidea from the Atlantic coast of North America. Proc. U. S. nation. Mus., 35: 71-86.

- 1911. Les Crustacés Isopodes du Travailleur et du Talisman. Formes nouvelles. Bull. Mus. Hist. nat. Paris, 7: 518-534.

Stephensen, K., 1915. Isopoda, Cumacea, Amphipoda, (excl. Hyperiidea). Rep. Danish oceanogr. Exped. Mediterranean, 2 (D1): 1-53.

WolfF, T., 1962. The systematics and biology of bathyal and abyssal Isopoda Asellota. Galathea Rep., 6: 1-315. 